\title{
LOCAI AND UNIFORM NEAR SMOOTHNESS OF SOME BANACH SPACES
}

\author{
LESZEK OLSZOWY
}

\begin{abstract}
In this paper we give an estimate of the modulus of near smoothness of the space $c_{0}\left(E_{i}\right)$. In the case of the space $c_{0}\left(l_{p i}\right)$ the exact formula for this modulus is derived. Moreover, we show that the properties of near uniform smoothness and local near uniform smoothness are hereditary with respect to the product space $c_{0}\left(E_{i}\right)$.
\end{abstract}

In the last years the notions being fundamental in the classical geometry of Banach spaces, such as smoothness and uniform smoothness have been translated in terms of the measure of noncompactness. This way came into existence a branch of the geometry of Banach spaces involving compactness conditions (cf. [1, 3, 8, 9, 10] and references therein).

The aim of this paper is to study a few concepts of the theory mentioned above. At the beginning we start with some notation.

Let $E$ be an infinite dimensional real Banach space with the dual $E^{*}$. Denote by $B$, $B^{*}, S, S^{*}$ the unit balls and the unit spheres in $E$ and $E^{*}$, respectively. For a bounded subset $X$ of $E$ let $\chi_{E}(X)$ denote the Hausdorff measure of noncompactness of $X$ defined as the infimum of all numbers $r>0$ such that $X$ can be covered by a finite family of balls with radii $r$.

Recall [1] that the modulus of near smoothness of the space $E$ is the function $\Sigma_{E}:[0,1] \rightarrow$ $[0,1]$ defined by the formula

$$
\Sigma_{E}(\varepsilon)=\sup \left\{\chi_{E^{*}}\left(F^{*}(x, \varepsilon)\right): x \in S\right\}
$$

where $F^{*}(x, \varepsilon)=\left\{f \in B^{*}: f(x) \geq 1-\varepsilon\right\}$.

Roughly speaking the modulus of near smoothness inform us about the noncompactness of the set of all hyperplanes supporting the unit sphere $S$ at an arbitrary point.

A space $E$ is called nearly uniformly smooth $(N U S)[1]$ whenever $\lim _{\varepsilon \rightarrow 0} \Sigma_{E}(\varepsilon)=0$. E is said to be nearly smooth $(N S)$ if $\Sigma_{E}(0)=0$. Moreover, a space $E$ is referred to as locally nearly uniformly smooth $(L N U S)$ [2] if $\lim _{\varepsilon \rightarrow 0} \chi_{E^{*}}\left(F^{*}(x, \varepsilon)\right)=0$ for every $x \in S$.

Let us pay attention to some results obtained before.

Received Auguest 23, 1995, revised May 20, 1997.

1991 Mathematics Subject Classification. 46B20.

Key words and phrases. Near uniform smoothness, modulus of near smoothness, measure of noncompactness, property $H^{*}$. 
In the case of the classical sequence spaces $c_{0}$ and $l_{p}(1<p<\infty)$ the following formulas can be derived [3] for $\varepsilon \in[0,1]$ :

$$
\begin{gathered}
\Sigma_{c_{0}}(\varepsilon)=\varepsilon \\
\Sigma_{l_{p}}(\varepsilon)=\left(1-(1-\varepsilon)^{q}\right)^{\frac{1}{q}}
\end{gathered}
$$

where $\frac{1}{p}+\frac{1}{q}=1$.

Thus both $c_{0}$ and $l_{p}$ are NUS spaces.

The example of the space $c_{0}$ shows simultaneously that a NUS space has not to be reflexive. The other facts in this direction can be found in $[1,2]$, for example.

The next concept which turns out to be connected with the properties defined above, is the property $H^{*}$. We say [2] that the norm $\|\cdot\|$ in a Banach space $E$ has the property $H^{*}$ whenever for any sequence $\left(f_{n}\right) \subset E^{*}$ converging weakly star to $f \in E^{*}$ and such that $\lim _{n \rightarrow \infty}\left\|f_{n}\right\|_{E^{*}}=\|f\|_{E^{*}}$ we have that $f_{n} \longrightarrow f$ in the norm of $E^{*}$.

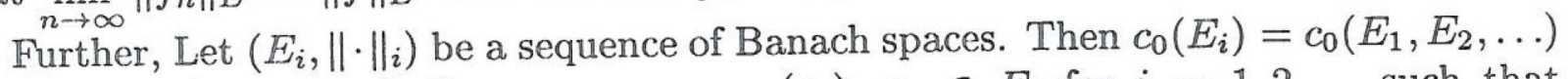
is the Banach space of all sequences $x=\left(x_{i}\right), x_{i} \in E_{i}$ for $i=1,2, \ldots$ such that $\lim _{n \rightarrow \infty}\left\|x_{i}\right\|_{i}=0$, furnished with the norm

$$
\left\|x_{0}\right\|=\max \left\{\left\|x_{i}\right\|_{i}: i \in \mathbb{N}\right\} .
$$

In the similar way we define the space $l_{p}\left(E_{i}\right)=l_{p}\left(E_{1}, E_{2}, \ldots\right)(1 \leq p<\infty)$ under the norm

$$
\|x\|_{p}=\left(\sum_{i=1}^{\infty}\left\|x_{i}\right\|_{i}^{p}\right)^{1 / p} .
$$

Let us mention that in $[2,3,5,6]$ it was shown that both the properties related to convexity such as $N S C, N U C, L N U C, H$ and the properties connected with smoothness as $N U S, L N U S, N S, H^{*}$ are hereditary with respect to the space $l_{p}\left(E_{i}\right)$. On the other hand it is easily seen that the space $c_{0}\left(E_{i}\right)$ is no longer $N S C, N U C, L N U C$ and it has no the property $\mathbb{H}$. Nevertheless, if we assume that $E_{i}$ is $N S(i=1,2, \ldots)$ then the space $c_{0}\left(E_{i}\right)$ has also this property (cf. [4]).

In the sequel we prove similar results for the "smothness" properties such as NUS, LNUS and $H^{*}$.

We start with the following theorem.

Theorem $\mathbb{1}$. Let $r(\varepsilon)=\sup \left\{\Sigma_{E_{i}}(\varepsilon): i \in \mathbb{N}\right\}$ for $\varepsilon \in[0,1]$. Then

$$
\Sigma_{c_{0}\left(E_{i}\right)}(\varepsilon) \leq r(\sqrt{\varepsilon})+2 \sqrt{\varepsilon}+2 \varepsilon .
$$

Proof. For convenience denote by $B, B^{*}, S, S^{*}$ the unit balls and the unit spheres in the spaces $c_{0}\left(E_{i}\right)$ and $\left(c_{0}\left(E_{i}\right)\right)^{*}=l_{1}\left(E_{i}^{*}\right)$, respectively. The norms in $E_{i}$ and $E_{i}^{*}$ will be denoted by $\|\cdot\|_{i}$ while $\|\cdot\|_{1}$ stands for the norm in $l_{1}\left(E_{i}^{*}\right)$.

Now, fix a number $\varepsilon \in(0,1]$ and take a number $\eta>0$ small enough. Choose $x=\left(x_{i}\right) \in S$ and a number $\gamma$ in such a way that

$$
\chi_{l_{1}\left(E_{i}^{*}\right)}\left(F^{*}(x, \varepsilon)\right)>\gamma>\Sigma_{c_{0}\left(E_{i}\right)}(\varepsilon)-\eta
$$


Then there exists a sequence $\left(f_{n}\right) \subset B^{*}$ satisfying the inequalities $1-\varepsilon \leq f(x)$ and $\gamma<\left\|f^{n}-f^{m}\right\|_{1}$ for $n, m \in \mathbb{N}, n \neq m$. Writting $f^{n}=\left(f_{i}^{n}\right)$, where $f_{i}^{n} \in E_{i}^{*}$ for every $i \in \mathbb{N}(n=1,2 \ldots)$ we can write the last inequalities in the form

$$
1-\varepsilon \leq \sum_{i=1}^{\infty} f_{i}^{n}\left(x_{i}\right) \quad \text { and } \quad \gamma<\sum_{i=1}^{\infty}\left\|f_{i}^{n}-f_{i}^{m}\right\|_{i}
$$

Applying the same argumentation as in [8] we may assume without loss of generality that

$$
\left\|f_{i}^{n}\right\|_{i} \longrightarrow a_{i}, \quad f_{i}^{n}\left(x_{i}\right) \longrightarrow b_{i}, \quad\left\|f_{i}^{n}-f_{i}^{m}\right\|_{i} \longrightarrow c_{i}
$$

when $n \longrightarrow \infty, m, n \longrightarrow \infty(i=1,2, \ldots)$.

Further, for $\delta>0$ consider the sets $S_{\delta}, T_{\delta}$ and $W_{\delta}$ defined below:

$$
\begin{aligned}
S_{\delta} & =\left\{i \in \mathbb{N}:\left\|x_{i}\right\|_{i}>\delta \quad \text { and } \quad a_{i}>0\right\} \\
T_{\delta} & =\left\{i \in \mathbb{N}:\left\|x_{i}\right\|_{i}>\delta \quad \text { and } \quad a_{i}=0\right\}, \\
W_{\delta} & =\left\{i \in \mathbb{N}:\left\|x_{i}\right\|_{i} \leq \delta\right\} .
\end{aligned}
$$

Obiously $\mathbb{N}=S_{\delta} \cup T_{\delta} \cup W_{\delta}$ in which $T_{\delta}$ is finite set. For $m \in \mathbb{N}$ large enough we have $\sum_{i \in T_{\delta}} f_{i}^{m}\left(x_{i}\right) \leq \delta$ and $\sum_{i \in W_{\delta}} f_{i}^{m}\left(x_{i}\right) \leq \delta$. This inequalities together with (3) yields $1-\varepsilon-2 \delta \leq \sum_{i \in S_{\delta}} f_{i}^{m}\left(x_{i}\right)$ for $m \in \mathbb{N}$ sufficiently large.

Hence, putting $S=\left\{i \in \mathbb{N}: x_{i} \neq \theta\right.$ and $\left.a_{i}>0\right\}$ and keeping in mind that $\cup_{\delta>0} S_{\delta}=S$, for $m \rightarrow \infty$ and $\delta \rightarrow 0$ we get

$$
1-\varepsilon \leq \sum_{i \in S} b_{i}
$$

Further, observe that

$$
1-\varepsilon \leq \sum_{i \in W_{\delta}} f_{i}^{m}\left(x_{i}\right)+\sum_{i \in \mathbb{N} \backslash W_{\delta}} f_{i}^{m}\left(x_{i}\right) \leq \delta \sum_{i \in W_{\delta}}\left\|f_{i}^{m}\right\|_{i}+\left(1-\sum_{i \in W_{\delta}}\left\|f_{i}^{m}\right\|_{i}\right) .
$$

This implies $\sum_{i \in W_{\delta}}\left\|f_{i}^{m}\right\|_{i} \leq \frac{\varepsilon}{1-\delta}$. Hence, in view of (3) we obtain

$$
\begin{gathered}
\gamma<\sum_{i \in W_{\delta}}\left\|f_{i}^{n}-f_{i}^{m}\right\|_{i}+\sum_{i \in S_{\delta}}\left\|f_{i}^{n}-f_{i}^{m}\right\|_{i}+\sum_{i \in T_{\delta}}\left\|f_{i}^{n}-f_{i}^{m}\right\|_{i} \leq \\
\leq \frac{2 \varepsilon}{1-\delta}+\sum_{i \in S_{\delta}}\left\|f_{i}^{n}-f_{i}^{m}\right\|_{i}+\sum_{i \in T_{\delta}}\left\|f_{i}^{n}-f_{i}^{m}\right\|_{i} .
\end{gathered}
$$

Using (4) and taking into account that $c_{i}=0$ for $i \in T_{\delta}$ we derive that

$$
\gamma-\frac{2 \varepsilon}{1-\delta} \leq \sum_{i \in S_{\delta}} c_{i}
$$


for $n, m \longrightarrow \infty$. Hence, letting $\delta \rightarrow 0$ we get

$$
\gamma-2 \varepsilon \leq \sum_{i \in S} c_{i}
$$

Now, let, us put $P=\left\{i \in S: \frac{b_{i}}{a_{i} \cdot\left\|x_{i}\right\|_{i}}>1-\sqrt{\varepsilon}\right\}$. Then the inequality (5) gives

$$
\begin{aligned}
1-\varepsilon & \leq \sum_{i \in S} b_{i}=\sum_{i \in P} b_{i}+\sum_{i \in S \backslash P} \frac{b_{i}}{a_{i}\left\|x_{i}\right\|_{i}} a_{i}\left\|x_{i}\right\|_{i} \\
& \leq \sum_{i \in P} b_{i}+(1-\sqrt{\varepsilon}) \sum_{i \in S \backslash P} a_{i}\left\|x_{i}\right\|_{i} \\
& \leq \sum_{i \in P} b_{i}+(1-\sqrt{\varepsilon})\left(\sum_{i \in S} a_{i}\left\|x_{i}\right\|_{i}-\sum_{i \in P} b_{i}\right) \\
& \leq \sum_{i \in P} b_{i}+(1-\sqrt{\varepsilon})\left(1-\sum_{i \in P} b_{i}\right) .
\end{aligned}
$$

Hence, after simple calculation we obtain $1-\sqrt{\varepsilon} \leq \sum_{i \in P} b_{i}$. Since $b_{i} \leq a_{i}$ we have $1-\sqrt{\varepsilon} \leq \sum_{i \in P} a_{i}$ and consequently

$$
\sum_{i \in S \backslash P} a_{i}=\sum_{i \in S} a_{i}-\sum_{i \in P} a_{i} \leq 1-(1-\sqrt{\varepsilon})=\sqrt{\varepsilon}
$$

what together with $c_{i} \leq 2 a_{i}$ implies $\sum_{i \in S \backslash P} c_{i} \leq 2 \sqrt{\varepsilon}$.

Further observe that by the inequality (7) we obtain

$$
\begin{gathered}
\sum_{i \in P} c_{i}=\sum_{i \in S} c_{i}-\sum_{i \in S \backslash P} c_{i} \geq \gamma-2 \varepsilon-2 \sqrt{\varepsilon} \quad \text { i.e. } \\
\sum_{i \in P} c_{i} \geq \gamma-2 \varepsilon-2 \sqrt{\varepsilon} .
\end{gathered}
$$

Notice, that the following two cases are possible:

(i) $\gamma-2 \varepsilon-2 \sqrt{\varepsilon} \leq 0$,

(ii) $\gamma-2 \varepsilon-2 \sqrt{\varepsilon}>0$.

In the case of (i) by (2) we get $\Sigma_{c_{0}\left(E_{i}\right)}(\varepsilon)<\eta+\gamma \leq \eta+2 \varepsilon+2 \sqrt{\varepsilon}$ which as $\eta \longrightarrow 0$ gives (1).

Now suppose that the case (ii) is satisfied and take $\delta>0$ small enough. Then, there exists $i \in P$ such that

$$
\frac{c_{i}}{a_{i}} \geq \gamma-2 \varepsilon-2 \sqrt{\varepsilon}-\delta>0
$$

Indeed, if not then $c_{i}<a_{i} \cdot(\gamma-2 \varepsilon-2 \sqrt{\varepsilon}-\delta)$ for every $i \in P$. 
This yields

$$
\sum_{i \in P} c_{i}<(\gamma-2 \varepsilon-2 \sqrt{\varepsilon}-\delta) \sum_{i \in P} a_{i} \leq \gamma-2 \varepsilon-2 \sqrt{\varepsilon}-\delta
$$

but this contradicts (8).

Thus let us take $i \in P$ satisfying (9) and put $\bar{x}=\frac{x_{i}}{\left\|x_{i}\right\|_{i}}, g^{n}=\frac{f_{i}^{n}}{\left\|f_{i}^{n}\right\|_{i}}$. By the definition of the set $P$ and (4) we have that $\lim _{n \rightarrow \infty} g^{n}(\bar{x})=\frac{b_{i}}{a_{i}\left\|x_{i}\right\|_{i}}>1-\sqrt{\varepsilon}$ what means that $F^{*}(\bar{x}, \sqrt{\varepsilon}) \supset\left\{g^{n}: n \geq k\right\}$ for some $k \in \mathbb{N}$.

Further using (4) and (9) we obtain

$$
\left\|g^{n}-g^{m}\right\|_{i} \longrightarrow \frac{c_{i}}{a_{i}} \geq \gamma-2 \varepsilon-2 \sqrt{\varepsilon}-\delta \text { when } \quad n, m \rightarrow \infty
$$

These facts imply

$$
r(\sqrt{\varepsilon}) \geq \chi_{E_{i}^{*}}\left(F^{*}(\bar{x}, \sqrt{\varepsilon})\right) \geq \chi_{E_{i}^{*}}\left(\left\{g^{n}: n \geq k\right\}\right) \geq \frac{c_{i}}{a_{i}} \geq \gamma-2 \varepsilon-2 \sqrt{\varepsilon}-\delta
$$

i.e. $\gamma \leq \delta+2 \varepsilon+2 \sqrt{\varepsilon}+r(\sqrt{\varepsilon})$. Combining the last inequality and (2) we infer

$$
\Sigma_{c_{0}\left(E_{i}\right)}(\varepsilon)<\eta+\gamma \leq \eta+\delta+2 \varepsilon+2 \sqrt{\varepsilon}+r(\sqrt{\varepsilon}),
$$

and the arbitrariness of $\eta$ and $\delta$ allows us to obtain (1).

Thus the proof is complete.

Corollary. The space $c_{0}\left(E_{i}\right)$ is NUS if and only if $\lim _{\varepsilon \rightarrow 0} r(\varepsilon)=0$.

Indeed, in view of the inequalities

$$
\Sigma_{E_{j}}(\varepsilon) \leq r(\varepsilon) \leq \Sigma_{c_{0}\left(E_{i}\right)}(\varepsilon) \text { for } j=1,2, \ldots
$$

we infer that if $c_{0}\left(E_{i}\right)$ is UNS then $\lim _{\varepsilon \rightarrow 0} r(\varepsilon)=0$. The converse implication is a consequence of Theorem 1.

Theorem 2. The space $c_{0}\left(E_{i}\right)$ is LNUS if and only if $E_{i}$ is LNUS for $i=1,2, \ldots$

Proof. The implication $\Longrightarrow$ is obvious. For the proof of the converse implication suppose contrary, i.e., there exists a number $\gamma>0$ and $x=\left(x_{i}\right) \in S$ such that $\lim _{\varepsilon \rightarrow 0} \chi\left(F^{*}(x, \varepsilon)\right)>\gamma>0$, where $\chi=\chi_{l_{1}\left(E_{i}^{*}\right)}$.

Let us fix $\delta>0$ such that

$$
\frac{\gamma}{2}<\gamma-\frac{2 \varepsilon}{1-\delta} \quad \text { for } \quad \varepsilon \in[0, \delta]
$$

Take $\varepsilon_{n} \in(0, \delta]$ with $\varepsilon_{n}$ monotone decreasing to zero. Repeating the argumentation in the Take $\varepsilon_{n} \in(0, \delta]$ with $\varepsilon_{n}$ mon of Theorem 1 we may assume that there exists a sequence $\left(f^{n}\right) \subset B^{*}, f^{n}=\left(f_{i}^{n}\right)$
proof of such that $1-\varepsilon_{n} \leq \sum_{i=1}^{\infty} f_{i}^{n}\left(x_{i}\right), \gamma<\sum_{i=1}^{\infty}\left\|f_{i}^{n}-f_{i}^{m}\right\|_{i}$ and $\left\|f_{i}^{n}\right\|_{i} \longrightarrow a_{i}, f_{i}^{n}\left(x_{i}\right) \longrightarrow b_{i}$, $\left\|f_{i}^{n}-f_{i}^{m}\right\|_{i} \longrightarrow c_{i}$ when $m, n \rightarrow \infty(i=1,2, \ldots)$. 
Lemma 1 . If $x_{i} \neq \theta$ and $a_{i}>0$ the $\chi_{E_{i}^{*}}\left(F^{*}\left(\frac{x_{i}}{\left\|x_{i}\right\|_{i}}, \frac{2 \varepsilon_{m}}{a_{i}\left\|x_{i}\right\|_{i}}\right)\right) \geq \frac{c_{i}}{2}, m=1,2, \ldots$

Proof. Observe that if $n>m$ then

$$
f_{i}^{n}\left(x_{i}\right) \geq\left\|f_{i}^{n}\right\|_{i} \cdot\left\|x_{i}\right\|_{i}-\varepsilon_{m} \quad \text { for } \quad i \in \mathbb{N} \text {. }
$$

Indeed, suppose the contrary, i.e., there exists $j \in \mathbb{N}$ such that $f_{j}^{n}\left(x_{j}\right)<\left\|f_{j}^{n}\right\|_{j} \cdot\left\|x_{j}\right\|_{j}-\varepsilon_{m}$. Then

$$
1-\varepsilon_{m} \leq 1-\varepsilon_{n} \leq f_{j}^{n}\left(x_{j}\right)+\sum_{i \neq j} f_{i}^{n}\left(x_{i}\right)<-\varepsilon_{m}+\sum_{i \in \mathbb{N}}\left\|f_{i}^{n}\right\|_{i} \cdot\left\|x_{i}\right\|_{i} \leq 1-\varepsilon_{m}
$$

which gives a contradiction.

Let $x_{i} \neq \theta$ and $a_{i}>0$. Put $g^{n}=\frac{f_{i}^{n}}{\left\|f_{i}^{n}\right\|_{i}}$. From (11) we have $g^{n}\left(\frac{x_{i}}{\left\|x_{i}\right\|_{i}}\right) \geq 1-\frac{\varepsilon_{m}}{\left\|f_{i}^{n}\right\|_{i} \cdot\left\|x_{i}\right\|_{i}}$ and for each large enough positive integer $n$ we have $g^{n}\left(\frac{x_{i}}{\left\|x_{i}\right\|_{i}}\right) \geq 1-\frac{2 \varepsilon_{m}}{a_{i}\left\|x_{i}\right\|_{i}}$. On the other hand $\left\|g^{n}-g^{m}\right\| \longrightarrow \frac{c_{i}}{a_{i}}$ when $n, m \longrightarrow \infty$. These yield $\chi_{E_{i}^{*}}\left(F^{*}\left(\frac{x_{i}}{\left\|x_{i}\right\|_{i}}, \frac{2 \varepsilon_{m}}{a_{i} \cdot\left\|x_{i}\right\|_{i}}\right)\right) \geq$ $\chi_{E_{i}^{*}}\left(\left\{g^{n}: n \in \mathbb{N}\right\}\right) \geq \frac{c_{i}}{2 a_{i}} \geq \frac{c_{i}}{2}$, and the proof of Lemma 1 is complete.

For what follows let us observe that (6) and (10) imply $\frac{\gamma}{2}<\sum_{i \in S_{\delta}} c_{i}$. From the last inequality we obtain that there exists $j \in S_{\delta}$ such that $\frac{\gamma}{2 p}<c_{j}$, where $p$ denotes the cardinality of the set $S_{\delta}$. For this $j$ we derive from Lemma 1 that

$$
\chi_{E_{j}^{*}}\left(F^{*}\left(\frac{x_{j}}{\left\|x_{j}\right\|_{j}}, \frac{2 \varepsilon_{m}}{a_{j} \cdot\left\|x_{j}\right\|_{j}}\right)\right) \geq \frac{c_{j}}{2}>\frac{\gamma}{4 p}>0,
$$

and by taking $m \longrightarrow \infty$ we get

$$
\lim _{\varepsilon \rightarrow 0} \chi_{E_{j}^{*}}\left(F^{*}\left(\frac{x_{j}}{\left\|x_{j}\right\|_{j}}, \varepsilon\right)\right)=\lim _{m \rightarrow \infty} \chi_{E_{j}^{*}}\left(F^{*}\left(\frac{x_{j}}{\left\|x_{j}\right\|_{j}}, \frac{2 \varepsilon_{m}}{a_{j} \cdot\left\|x_{j}\right\|_{j}}\right)\right) \geq \frac{\gamma}{4 p}>0 .
$$

But this contradicts to the assumption that the spaces $E_{i}$ have the property $L N U S$ and completes the proof.

Theorem 3. The space $c_{0}\left(E_{i}\right)$ has property $H^{*}$ if and only if the spaces $E_{i}$ have this property $(i=1,2, \ldots)$.

Proof. Suppose that $E_{i}$ has property $H^{*}$ for $i=1,2, \ldots$ Take arbitrary sequence $\left(f^{n}\right)=\left(f_{j}^{n}\right) \subset l_{1}\left(E_{i}^{*}\right)$ which is weakly star convergent to some $f=\left(f_{i}\right) \in l_{1}\left(E_{i}^{*}\right)$ and such that $\lim _{n \rightarrow \infty}\left\|f^{n}\right\|_{1}=\|f\|_{1}$. We have to prove that $\lim _{n \rightarrow \infty} \sum_{i=1}^{\infty}\left\|f_{i}^{n}-f_{i}\right\|_{i}=0$. Let $\left(g^{n}\right)$ be an arbitrary subsequence of $\left(f^{n}\right)$. By the diagonal procedure we may select a subsequence $\left(h^{n}\right)=\left(h_{i}^{n}\right)$ of $\left(g^{n}\right)$ such that

$$
\left\|h_{i}^{n}\right\|_{i} \longrightarrow a_{i} \text { when } n \longrightarrow \infty \text {. }
$$


Obviously $\lim _{n \rightarrow \infty}\left\|h^{n}\right\|_{1}=\|f\|_{1}$ what means

$$
\lim _{n \rightarrow \infty} \sum_{i=1}^{\infty}\left\|h_{i}^{n}\right\|_{i}=\sum_{i=1}^{\infty}\left\|f_{i}\right\|_{i}
$$

If we fix $j \in \mathbb{N}$ we have $\sum_{i=1}^{j}\left\|h_{i}^{n}\right\|_{i} \leq \sum_{i=1}^{\infty}\left\|h_{i}^{n}\right\|_{i}$, and when $n \longrightarrow \infty$ we get from (12) and (13) that $\sum_{i=1}^{j} a_{i} \leq \sum_{i=1}^{\infty}\left\|f_{i}\right\|_{i}$. This implies

$$
\sum_{i=1}^{\infty} a_{i} \leq \sum_{i=1}^{\infty}\left\|f_{i}\right\|_{i}
$$

On the other hand, because $h^{n}=\left(h_{1}^{n}, h_{2}^{n}, \ldots\right)$ converges weakly star to $f=\left(f_{1}, f_{2}, \ldots\right)$ we infer that $\left(h_{i}^{n}\right)$ converges weakly star to $f_{i}$ for $i=1,2, \ldots$ which gives $\left\|f_{i}\right\|_{i} \leq \liminf _{n \rightarrow \infty}\left\|h_{i}^{n}\right\|_{i}$ i.e. $\left\|f_{i}\right\|_{i} \leq a_{i}$. The last inequality together with (14) yields $\left\|f_{i}\right\|_{i}=a_{i}=\lim _{n \rightarrow \infty}\left\|h_{i}^{n}\right\|_{i}$ for $i=1,2, \ldots$ and from the property $H^{*}$ for $E_{i}$ we obtain

$$
\lim _{n \rightarrow \infty}\left\|h_{i}^{n}-f_{i}\right\|_{i}=0 \text { for } i=1,2, \ldots
$$

Further, let us fix $\varepsilon>0$ and take $k \in \mathbb{N}$ so large that

$$
\sum_{i=k+1}^{\infty}\left\|f_{i}\right\|_{i} \leq \frac{\varepsilon}{5}
$$

Keepping in mind (15) and (13) we may choose $m \in \mathbb{N}$ so large that

$$
\sum_{i=1}^{k}\left\|h_{i}^{n}-f_{i}\right\|_{i} \leq \frac{\varepsilon}{5}
$$

and $\sum_{i=1}^{\infty}\left\|h_{i}^{n}\right\|_{i}-\sum_{i=1}^{\infty}\left\|f_{i}\right\|_{i} \leq \frac{\varepsilon}{5}$ for $n \geq m$

The last inequality gives

$$
\sum_{i=1}^{k}\left(\left\|h_{i}^{n}\right\|_{i}-\left\|f_{i}\right\|_{i}\right)+\sum_{i=k+1}^{\infty}\left(\left\|h_{i}^{n}\right\|_{i}-\left\|f_{i}\right\|_{i}\right) \leq \frac{\varepsilon}{5} .
$$

Hence, by the inequalities (17) and (16) we obtain

$$
\begin{aligned}
& \sum_{i=k+1}^{\infty}\left\|h_{i}^{n}\right\|_{i} \leq \sum_{i=k+1}^{\infty}\left\|f_{i}\right\|_{i}+\left|\sum_{i=1}^{k}\left(\left\|h_{i}^{n}\right\|_{i}-\left\|f_{i}\right\|_{i}\right)\right|+\frac{\varepsilon}{5} \leq \frac{\varepsilon}{5} \\
& +\sum_{i=1}^{k}\left\|h_{i}^{n}-f_{i}\right\|_{i}+\frac{\varepsilon}{5} \leq \frac{\varepsilon}{5}+\frac{\varepsilon}{5}+\frac{\varepsilon}{5}=\frac{3 \varepsilon}{5} \quad \text { for } \quad n \geq m
\end{aligned}
$$


Linking the last inequality, (17) and (16) we derive

$$
\begin{aligned}
& \sum_{i=1}^{\infty}\left\|h_{i}^{n}-f_{i}\right\|_{i} \leq \sum_{i=1}^{k}\left\|h_{i}^{n}-f_{i}\right\|_{i}+\sum_{i=k+1}^{\infty}\left(\left\|h_{i}^{n}\right\|_{i}-\left\|f_{i}\right\|_{i}\right) \\
& \leq \frac{\varepsilon}{5}+\frac{3 \varepsilon}{5}+\frac{\varepsilon}{5}=\varepsilon \text { for } n \geq m,
\end{aligned}
$$

which means that $\left(h^{n}\right)$ converges to $f$ in the norm of the space $l_{1}\left(E_{i}^{*}\right)$. The arbitrariness of the subsequence $\left(g^{n}\right)$ implies the same for $\left(f^{n}\right)$.

This completes the proof as the converse implication is obvious.

Example. Let us take the spaces $l_{p_{i}}, p_{i}>1, i=1,2, \ldots$ and put $\bar{q}=\sup \left\{q_{i}: i \in \mathbb{N}\right\}$, where $\frac{1}{p_{i}}+\frac{1}{q_{i}}=1$. We prove that if $\bar{q}<\infty$ then

$$
\Sigma_{c_{0}\left(l_{p_{i}}\right)}(\varepsilon)=\left(1-(1-\varepsilon)^{\bar{q}}\right)^{\frac{1}{\bar{q}}} .
$$

In the case $\bar{q}=\infty$ we show that

$$
\Sigma_{c_{0}\left(l_{p_{i}}\right)}(\varepsilon)=\left\{\begin{array}{l}
0 \text { for } \varepsilon=0, \\
1 \text { for } \varepsilon \in(0,1] .
\end{array}\right.
$$

Proof. In what follows we will use the inequality

$$
\left(\sum_{k=1}^{\infty} s_{k}^{\frac{1}{\omega_{k}}}\right)^{\omega}+\left(\sum_{k=1}^{\infty} t_{k}^{\frac{1}{\omega_{k}}}\right)^{\omega} \leq\left(\sum_{k=1}^{\infty}\left(s_{k}+t_{k}\right)^{\frac{1}{\omega_{k}}}\right)^{\omega}
$$

where $1<\omega_{k} \leq \omega$ and $s_{k}, t_{k} \geq 0$ for $k=1,2, \ldots$, which is a consequence of a reasoning similar to the proof of Minkowski inequality.

We will also need the following Lemma.

Lemma 2. [5]. Let $E$ be a space with Schauder basis $\left(e_{n}\right)$ and let $R_{n}$ be the $n$ remainder operator $R_{n}\left(\sum_{i=1}^{\infty} \alpha_{i} e_{i}\right)=\sum_{i=n+1}^{\infty} \alpha_{i} e_{i}$.

Denote by $\left\|R_{n}\right\|$ the norm of the operator $R_{n}$. If $\left\|R_{n}\right\|=1$ for $n=1,2, \ldots$ then

$$
\chi(X)=\limsup _{n \rightarrow \infty}\left(\sup \left\{\left\|R_{n} x\right\|: x \in X\right\}\right) \quad \text { for } \quad X \subset E .
$$

In what follows denote by $\|\cdot\|_{1}$ the norm of the space $\left(c_{0}\left(l_{p_{i}}\right)\right)^{*}=l_{1}\left(l_{q_{i}}\right)$.

Let $f \in l_{1}\left(l_{q_{i}}\right)$. We describe $f=\left(f^{i}\right)=\left(f_{j}^{i}\right)$, where $f^{i} \in l_{q_{i}}, f_{j}^{i} \in \mathbb{R}$. Denote by $e_{n, k}$ the natural basis in $l_{1}\left(l_{q_{i}}\right)$, i.e.,

$$
\left(e_{n, k}\right)_{j}^{i}=\left\{\begin{array}{l}
1 \text { for } i=n \text { and } j=k \\
0 \text { for } i \neq n \text { or } j \neq k .
\end{array}\right.
$$

Further, let $h$ denotes one-to-one mapping between $\mathbb{N}$ and $\mathbb{N} \times \mathbb{N}$. 
Put

$$
e_{n}=e_{h(n)} .
$$

It is easy to check that $\left(e_{n}\right)$ is the Schauder basis in $l_{1}\left(l_{q_{i}}\right)$ and $\left\|R_{n}\right\|=1$.

We prove now the following Lemma.

Lemma 3. If $\bar{q}<\infty$ and $\left(e_{n}\right)$ is the basis in $l_{1}\left(l_{q_{i}}\right)$ defined in (22) then

$$
\left\|R_{n} f\right\|_{1}^{\bar{q}}+\left\|\left(I-R_{n}\right) f\right\|_{1}^{\bar{q}} \leq\|f\|_{1}^{\bar{q}}
$$

for $f \in l_{1}\left(l_{q_{i}}\right)$ and $n \in \mathbb{N}$.

Proof. Fix $n \in \mathbb{N}$ and $f=\left(f^{i}\right)=\left(f_{j}^{i}\right) \in l_{1}\left(l_{q_{i}}\right)$. Put $J_{k}=\{i \in \mathbb{N}:(k, i) \in$ $h(\{1,2, \ldots, n\})\}$. Applying the inequality (20) for $s_{k}=\left(\sum_{i \in J_{k}}\left|f_{i}^{k}\right|^{q_{k}}\right), t_{k}=\left(\sum_{i \in \mathbb{N} \backslash J_{k}}\left|f_{i}^{k}\right|^{q_{k}}\right)$, $\omega_{k}=q_{k}, \omega=\bar{q}$, and keeping in the mind that $\left\|R_{n} f\right\|_{1}=\sum_{k=1}^{\infty}\left(\sum_{i \in \mathbb{N} \backslash J_{k}}\left|f_{i}^{k}\right|^{q_{k}}\right), \|(I-$ $\left.R_{n}\right) f \|_{1}=\sum_{k=1}^{\infty}\left(\sum_{i \in J_{k}}\left|f_{i}^{k}\right|^{q_{k}}\right)^{\frac{1}{q_{k}}}$ and $\|f\|_{1}=\sum_{k=1}^{\infty}\left(\sum_{i=1}^{\infty}\left|f_{i}^{k}\right|^{q_{k}}\right)^{\frac{1}{q_{k}}}$ we derive (23), which finishes the proof of our lemma.

Now, let u suppose that $\bar{q}<\infty$. Take $\delta>0, x=\left(x^{i}\right)=\left(x_{j}^{i}\right) \in S_{c_{0}\left(l_{p_{i}}\right)}$ and $f=\left(f^{i}\right)=\left(f_{j}^{i}\right) \in F^{*}(x, \varepsilon)$. Choose $n_{0} \in \mathbb{N}$ which satisfies $\max \left\{\left\|x_{i}\right\|_{i}: i \geq n_{0}+1\right\} \leq \delta$. Further observe that there exists $m_{0} \in \mathbb{N}$ such that

$$
\left(\sum_{j=m_{0}+1}^{\infty}\left|x_{j}^{i}\right|^{p_{i}}\right)^{\frac{1}{p_{i}}} \leq \frac{\delta}{n_{0}} \text { for } i=1,2, \ldots, n_{0} .
$$

This implies

$$
\begin{aligned}
1-\varepsilon \leq & f(x) \leq \sum_{i=1}^{n_{0}} f^{i}\left(x^{i}\right)+\sum_{i=n_{0}+1}^{\infty}\left\|f^{i}\right\|_{i} \cdot\left\|x^{i}\right\|_{i} \leq \sum_{i=1}^{n_{0}}\left(\left(\sum_{j=1}^{m_{0}}\left|f_{j}^{i}\right|^{q_{i}}\right)^{\frac{1}{q_{i}}} \cdot\right. \\
& \left.\cdot\left(\sum_{j=1}^{m_{0}}\left|x_{j}^{i}\right|^{p_{i}}\right)^{\frac{1}{p_{i}}}\right)+\sum_{i=1}^{n_{0}}\left(\sum_{j=m_{0}+1}^{\infty}\left|f_{j}^{i}\right|^{q_{i}}\right)^{\frac{1}{q_{i}}} \cdot \frac{\delta}{n_{0}}+\delta \\
\leq & \sum_{i=1}^{n_{0}}\left(\sum_{j=1}^{m_{0}}\left|f_{j}^{i}\right|^{q_{i}}\right)^{\frac{1}{q_{i}}}+2 \delta \text { i.e. } \\
1-\varepsilon-2 \delta \leq & \sum_{i=1}^{n_{0}}\left(\sum_{j=1}^{m_{0}}\left|f_{j}^{i}\right|^{\frac{1}{q_{i}}}\right)^{\frac{1}{q_{i}}} .
\end{aligned}
$$


Let us find $n_{1} \in \mathbb{N}$ such that $h\left(\left\{1,2, \ldots, n_{1}\right\}\right) \supset\left\{1, \ldots, n_{0}\right\} \times\left\{1, \ldots, m_{0}\right\}$. This inclusion and the previous inequality yield

$$
1-\varepsilon-2 \delta \leq \sum_{i=1}^{n_{0}}\left(\sum_{j=1}^{m_{0}}\left|f_{j}^{i}\right|^{q_{i}}\right)^{\frac{1}{q_{i}}} \leq\left\|\left(I-R_{n}\right) f\right\|_{1} \quad \text { for } n \geq n_{1} .
$$

Hence, in the light of $(23)$ we obtain $\left\|R_{n} f\right\|_{1} \leq\left(1-(1-\varepsilon-2 \delta)^{\bar{q}}\right)^{\frac{1}{q}}$. for $f \in F^{*}(x, \varepsilon)$ and $n \geq n_{1}$ which by (21) implies

$$
\chi_{l_{1}\left(l_{q_{i}}\right)}\left(F^{*}(x, \varepsilon)\right)=\limsup _{n \rightarrow \infty}\left(\sup \left\{\left\|R_{n} f\right\|_{1}: f \in F^{*}(x, \varepsilon)\right\}\right) \leq\left(1-(1-\varepsilon-2 \delta)^{\bar{q}}\right)^{\frac{1}{\bar{q}}} .
$$

Consequently, in view of the arbitrariness of $\delta$ and $x \in S_{c_{0}\left(l_{p_{i}}\right)}$ we get $\Sigma_{c_{0}\left(l_{p_{i}}\right)}(\varepsilon) \leq$ $\left(1-(1-\varepsilon)^{\bar{q}}\right)^{\frac{1}{\bar{q})}}$. On the other hand

$$
\Sigma_{c_{0}\left(l_{p_{i}}\right)}(\varepsilon) \geq \sup \left\{\Sigma_{l_{p_{i}}}(\varepsilon)=\left(1-(1-\varepsilon)^{q_{i}}\right)^{\frac{1}{q_{i}}}: i \in \mathbb{N}\right\}=\left(1-(1-\varepsilon)^{\bar{q}}\right)^{\frac{1}{\bar{q}}}
$$

and (18) is proved.

In the case $\bar{q}=\infty$ we derive

$$
1 \geq \Sigma_{c_{0}\left(l_{p_{i}}\right)}(\varepsilon) \geq \sup \left\{\Sigma_{l_{p_{i}}}(\varepsilon)=\left(1-(1-\varepsilon)^{q_{i}}\right)^{\frac{1}{q_{i}}}: i \in \mathbb{N}\right\}=1,
$$

for $\varepsilon \in(0,1]$. Because the property $N S$ of space $E_{i}$ is transfered to $c_{0}\left(E_{i}\right)$ and $l_{p_{i}}$ is $N S$ then $c_{0}\left(l_{p_{i}}\right)$ is NS i.e. $\Sigma_{c_{0}\left(l_{p_{i}}\right)}(0)=0$ what gives $(19)$.

This ends the proof.

\section{References}

[1] J. Banaś and K. Fraczek, "Conditions involving compactness in geometry of Banach spaces," Nonlinear Analysis, 20(1993), 1217-1230.

[2] J. Banaś and K. Fraczek, "Locally nearly uniformly smooth Banach spaces," Collect. Math., 44(1993), 13-22.

[3] J. Banaś, "Compacness conditions in geometric theory of Banach spaces," Nonlin. Anal., T.M.A., 16(1991), 669-682.

[4] J. Banaś and K. Sadarangani, "Near smoothness of Banach spaces," Collect. Math., 46(1995), 279-287.

[5] J. Banaś and K. Goebel, "Measures of noncompacness in Banach spaces," Lecture Notes in Pure and Aplied Mathematics, 60(1980), Marcel Dekker, New York.

[6] I. E. Leonard, "Banach sequences spaces," J. Math. Analysis Aplic., 54(1976), 245-265.

[7] L. Olszowy, "The modulus of near smoothness of the $l^{p}$ product of a sequences of Banach spaces," Glasgow Math. J., 39(1997), 153-165.

[8] J. R. Partington, "On nearly uniformly convex Banach spaces," Math. Proc. Comb. Phil. Soc., 93(1983), 127-129.

[9] S. Prus, "Nearly uniformly smooth Banach spaces," Boll. Un. Math. Ital. (7), 3-B(1989), 507-512. 
[10] S. Prus, "On the modulus of noncompact convexity in a Banach space," Arch. Math. (Basel), 63(1994), 441-448.

Department of Mathematics, Technical University of Rzeszów, 35-959 Rzeszów, W. Pola 2, Poland.

E-mail: lolszowy@prz.rzeszow.pl 